theory offered by Erichsen, of the chief cause of the stagnation of blood in the capillaries of the lungs-viz., that the venous blood acting as a stimulant on the smaller arterial branches (considering the pulmonary veins as arteries, because they carry venous blood) cause their gradual tonic contraction, by which congestion of the systemic arteries and of the pulmonary arteries and capillaries ensues.*

I therefore suppose that the stimulant of venous blood acting on the arteries, and especially their extremities, causes their contraction, and thus produces general arterial congestion; whilst the left side of the heart, acting more feebly than usual, cannot overcome this, to transmit an impulse to the smaller arteries, as the radial or temporal, although it may be able to do so to the femoral or other large branch.

A further proof of some obstruction existing in the artery be yond the seat of puncture may be adduced from the action of the heart, which, during the intensity of the paroxysm, was diminished, yet the velocity with which the stream flowed from the orifice was not lessened ; indeed, it appeared to me to be rather increased; and Erichsen states that the mercury in a hæmadynamometer attached to an artery of a dog, rose from five and a half to five and three-quarters or six and a half inches in two minutes after the trachea was tied, rising higher after the animal bad ceased struggling than it did during that time; whilst another attached to a vein indicated a consiaerable diminution of pressure in its walls. When an animal was previously pithed, the same occurred.

But does carbonic acid act as a stimulant to the arteries? That arteries possess a contractile power, which may be excited by stimulants, few will, I think, deny, though many will not allow carbonic acid to have any such power. We know, that although carbonic acid acts as a sedative to the stomach, it is a powerful stimulant to the glottis, causing instant contraction of the orifice, though to produce this the carbonic acid must be but little diluted. $\dagger$ Wherefore, then, should we deny that it may act in a similar manner on the coats of the arteries? A further objection may be offered to this-viz., that we thus assert oxygen to be a sedative to the arteries, whilst it is a stimulant to all other parts; but a little consideration will show that it never was intended to act as a stimulant to their coats, but only on the organic processes proceeding in them; for if it did so, the passage of arterial blood through these canals would be stayed by the contraction induced, and the object for which they were formed would be defeated. We might as well expect that healthy bile would act as a stimulant to the healthy gall-bladder, totally independent of the quantity contained therein, and yet it produces most violent irritation of any other vital parts, except those through which it naturally passes. We must, therefore, consider that arterial blood does not increase the function of the coats of the arteries, and thus act as a stimulant, any more than a secretion excites its proper receptacle, independently of the bulk contained within it. More might be urged on the same subject, but space will not admit of it.

But it may be said, that even allowing this, there is no proof that the contraction occurs in the arteries and not in the capillaries. Erichsen adduces many arguments against the latter opinion, but I think his microscopic experiment sufficiently conclusive, and will therefore repeat it:- " On asphyxiating a young animal, a portion of whose mesentery has been fixed under a microscope, the following phenomena will ensue:-For about a minute after the struggles of the animal have ceased, the circulation appears to proceed with its usual rapidity; it then gradually becomes somewhat slower, the arteries contracting in size, containing less blood, and assuming a lighter and more tawny colour than before; whilst the veins become congested and fuller, assuming a beautifil crimson hue. As the circulation continues, the arteries acquire a lighter colour; the diminution in size, and the difference in the quantity of blood contained in them and the veins most marked." $f$ On artificial respiration being performed, these phenomera subsided.

If this theory be confirmed, it will be useful in explaining some of the secondary diseases which arise in those cases uhere the due arterialization of the blood is interfered with. Thus, dilatation of the right side of the heart, \&c. ; also, the action of medicines in the removal of tumours, new deposits, \&c.; as we well know that all new growths are affected by constitutional causes to a much greater extent than the old: thus a medicine pro. ducing a state of contraction of the arteries to an extent only sufficient to increase their activity, would probably act so intensely on those supplied to new growths, as to prevent a due

- Erichsen on Asphyxia, Edinburgh Medical and Surgical Journal, January, 1845 . supply of blood to them, and thus allow the absorbent to excee 1 the nutritive function.

The only remaining observations I would make in this case, consist in the alterations observed in the pulse: during the curative state it was weak, irregular, and laboured; in the first oceurrence of the convulsions it rose in power and falness, then became gradually imperceptible during the most intense part of the convulsions; then again gradually recovered its power. These changes were so invariable, that the on-coming of a paroxysm could be foretold by the increased strength of the pulse.

Commercial-road, March, 1845 .

\section{CASE OF STRANGULATED INGUINAL HERNIA REQUIRING} OPERATION.

By George Botromlky, Esq. Surgeon to the Croydon Infirmary. MARY —, aged thirty-four, mother of eight children, and pregnant with the ninth, had been the subject of right inguinal hernia, and wore a truss for about two years. She was seized on Monday with violent abdominal pains, extending to the back, accompanied with severe faintings; pulse small, quick, and irritable. Being in the third month of pregnancy, the pains were supposed to arise from uterine irritation; her bowels were open, and she was free from pain in the hernial tumour, which was stated to be quite reduced. On Tuesday, the symptoms increasing in urgency, an examination of the hernial tumour took place, when it was discovered that a small portion of its contents had descended below the pad of the truss, which pressed upon it; an immediate attempt was made to reduce it, without success. The usual remedial measures, such as hot bath, bleeding, cold applications, \&c., were had recourse to, without benefit. 'The symptoms inereasing in urgency, rendered it necessary that an operation should be performed; it was proposed early on the Wednesday mo"ning, but met with most decided opposition both from herself and her husband, although every persuasive means was used to obtain their consent ; but on Friday morning, when every ray of hope that the operation would succeed was gone, a request that it should be performed was made, the abdomen at the time being quite tympanitic, hiccup, vomiting of focal matter, sunken and distressed countenance, pulse thready and past counting, accompanied with cold perspiration; in point of fact, she appeared fast sinking, but with a view of giving her the most remote chance, I performed the operation in the usual way. Upon opening the sac, the escape of fluid was immense; the sac contained a knuckle of intestine, of a very dark colour, with several suspicious spots upon its surface, but as it did not appear to have totally lost its vitality, I returned it into the cavity of the abdomen; the wound was dressed, and the patient placed in bed, in an almost lifeless state. But reaction soon followed; leeches and blisters were applied to the abdomen, cathartic injections administered, and a dose of castor oil and opium given, which in a few hours produced some healthy alvine evacuations, and from that time she gradually and perfectly recovered.

In this case there are two remarkable features worthy of observation. In the first place, the intestine being strangulated for so long a time, the operation being performed when the patient was almost in a dying state, (the delay arising from consent not being given by the patient,) shows that it is our duty to perform operations under these circumstances, when even all chance of success is apparently gone. Her being in a state of pregnancy, and undergoing severe sufferings before the operation, and the shock of the operation not interfering with utero-gestation, are also circumstances which are worthy of being noted. She went on to her full period, gave birth to a very large and healthy child, and is at the present moment enjoying good health.

June 23rd, 1845.

\section{CASE OF OBSCURE ABNORMAL PULSATION IN THE ABDOMEN,} TREATED WITH TONICS

By A. WoLrF, Esq. Surgeon, London.

ON the 15 th of October I was called, rather suddenly, to visit Miss $\mathbf{L}-$, in St. Mary Axe. On arriving, I found a young lady, about twenty-four years of age, dark complexioned, with black hair and eyes. Her friends state that she generally enjoys good health, with the exception of occasional hysterical attacks. She has been ailing for the last few days, complaining of a dull pain abont the abdomen, and frequent faintings ; catamienia regular; bowels inclined to be costive; tongue rather coated; pulse small and quick.-An aperient draught and calomel pill to be taken directly; an antispasmodic mixture to be partaken of every four hours.

16th.-The aperient has acted copiously, the evacuations rather dark-coloured, in other respects of a healthy character. The pain of the abdomen has increased in intensity, and is accompanied by a sensation of throbbing. - Eight leeches to be applied 
to the abdomen. To continue the mixture. The aperient to be repeated.

17th.-The bowels have been freely opened. The leeches abstracted a considerable quantity of blood; their application was followed by a prolonged fainting fit. The pain is increased; the pulsation has also increased, and can now be distinctly felt by the hand applied just below the umbilicus; lips colourless; countenance very anxious. The patient states that for some time past she has felt an indistinct throbbing sensation about the abdomen.

(The friends having become much alarmed, it was deemed expedient to have the opinion of a physician. Dr. C-_ accordingly saw the patient in the afternoon. After a careful and prolonged examination he gave a very guarded prognosis, and ordered a plaster of belladonna extract to be applied over the abdomen. The mixture to be continued. An anodyne to be administered at night.)

18th.-The pulsation is increased in intensity, and can now be seen to move the coverings of the bed. The stethoscope being applied, the head of the auscultator is moved by the pulsation in a manner sufficiently evident to a person placed at some distance from the bed; there is extreme prostration; the bowels have been copiously and frequently evacuated. The action of the heart is feeble, and accompanied by a slight abnormal bruit; extremities cold. The pulsation is strictly confined to a space of about four inches, from one inch above the umbilicus down wards. Dr. C_- considers the case to be one of aneurism, and gives a decidedly unfarourable prognosis. The age of the patient, the somewhat sudden nature of the (aggravated symptoms of the) attack, with its uncommonly rapid progress, making me feel unconvinced of its being a case of aneurism, I requested that another surgeon should be consulted.

20th.-Mr. Aston Key accordingly saw the paticnt this day, and considered the case to be decidedly not one of aneurism, but that the pulsation depended on a peculiar state of the artery, caused by general derangement of the health. A course of tonic medicines, with occasional aperients, was prescribed.

This opinion proved to be correct; the patient gradually improved in health, and the ahnormal pulsation having as gradually diminished in intensity, ceased at the end of about five weeks. Miss L _ - has since been perfectly well. I am aware that several similar cases to the above have been recorded, and that they have been mistaken for aneurisms, by physicians and surgeons of the very highest reputation. Indeed, with a person who, for the first time, sees such a case, it must be very difficult to conceive that any cause, excepting considerable and permanent dilatation of a huge arterial trunk, could produce such violent and persistent pulsation, whilst the rest of the arterial system is in a tranquil state.

It affords also a useful and curious suhject for inquiry, (for it has not yet been explained,) What is the exact state of the artery during these attacks?

The record of such cases is further likely to be useful in impressing on the mind of the practitioner how rery cautiously he should receive (apparently incontrovertible) evidence of organic arterial disease. For persistent and abnormal pulsation, with a morbid sound, heard on the application of the stethoseope to the heart, has betrayed many practitioners into the error of mistaking for, and diagnosing as, organic disease, what eventually proves to have been only functional derangement.

Shoreditch, July Ist, 1845.

CASE OF IMPERFORATE HYMEN.

By Thomas G. Vawdrey, Esq., M.R.C.S., \&c, st. Anstell.

ON the 5th of April, 1844, I was requested by the parents to visit and prescribe for A. B-. I found her a well-grown young person, aged seventeen years and a half, of florid complexion and healthy appearance, in every particular well developed, and presenting the general appearance of having reached the period of puberty. She complained of severe forcing pains in the lower part of the back and abdomen, closely resembling labour pains ; the abdomen was swollen, but neither tender nor tympanitic; the fundus of the uterus could be distinctly felt at the unbilicus; the catamenia had never appeared. Under these circumstances, I considered it requisite to make an examination per vaginam, and found a firm, tense membrane completely closing up the orifice of the vagina, slightly protruding, and at first resembling the protrusion of the membranes. I at once marle a free incision into this membrane, which was immediately followed by the forcible discharge of about two quarts of a black-looking sanguineous fluid, not mixed with coagula, and free from any disagreeable odour. A piece of oiled lint was then inserted in the opening, to be removed and a fresh piece inserted daily. On the second day, an arerient was given, and the patient progressed farourably until the eighth day, when symptoms closely resembling puerperal peritonitis came on. 'The discharge from the vagina, which had continued up to this time, suddenly ceased; the pulse became quick and hard; the abdomen tender, \&c. Bleeding from the arm, and by a large number of leeches to the abdomen, with calomel and opium internally, soon subdued these symptoms. As soon as the gums were effected by the calomel, the discharge from the vagina returned, and the patient's recovery progressed favourably. It is satisfactory to add that, up to the present time, the catamenia appeared at the regular periods, and the young lady's health has continued in every respect good.

Cornwall, July 2nd, 1845 .

PREGNANCY, OCCURRING WITH (APPARENTLY) COMPLETE. OCCLUSION OF THE VAGINA.

By C. CARTer, Esq. M.D., Montreal.

ON the 25th of April, 1845, I was requested to visit Mrs. Sa delicate dyspeptic woman, aged thirty, in labour with her second child. She had suffered much in her previous confinement, (having been delivered by instruments,) and recovered slowly. On examination, I perceived that there had been extensive laceration of the perineum, the vagina and rectum having been made one sulcus. Following the direction of the vagina, about three inches from its orifice, the finger was arrested in a pointed cul de sac; withdrawn about an inch and a half, and directed backwards, it entered the rectum, the anterior edge of which was here sharp and thin, but surrounded a little higher up by distinct muscular fibres, which, when irritated by the finger, contracted, supplying, in some measure, the place of the sphincter. The os uteri could not be detected cither by the rectum or through the walls of the cul de sac. A very careful examination was now made with a silver catheter by myself and a friend, to discover, if possible, any foramen or canal which the finger could not detect, but none existed. During a pain, I distinctly ascertained the presence of fluid behind the fleshy septum, and cautiously introduced a bistoury, which immediately produced a gush of liquor amnii. A couple of fingers were now introduced into the cavity of the uterus, and the head of the child was found presenting, but situated above the brim of the pelvis, which was unusually narrow both in its lateral and antero-posterior diameters. At three, P.M., found the head much lower in the pelvis, but tightly pncircled by the rigid os nteri and remains of the vagina, which tension I could only relieve by several slight incisions with a bistoury around the circumference. Anteriorly, the head was impeded by a fleshy mass, which proved to be the bladder displaced and thrown forwards in the former labour. The application of the forceps, rendered indispensable by the contracted state of the pelvis, was attended with much difficulty. The pains now became very powerful, but from the state of impaction it required considerable and repeated efforts on my part to effect the delivery with safety. After waiting an hour and a half I was obliged to extract the placenta, and experienced some difficulty in introducing my hand, in consequence of the narrowness of the pelvis between the ramii. The woman, although very weak, soon rallied, and both she and the child appeared yesterday to be doing well.

April 2sth, 1845.

** The above case has been sent to us for publication by Mr. John Coward, Surgeon, of Minerva-terrace, Islington.ED. L.

PERIOSTIC TUMOUR, THE RESULT OF STUMPS OF TEETH REMAINING IN THE JAW.

By H. C. VANDERPANT, Esq. Surgeon-Dentist, London.

A FEw months ago, I had under my care a case of severe disease of the upper jaw, produced by caries of the fangs of teeth, which the patient had neglected to have extracted, from apprehension of the suffering.

The patient was a female, aged forty-two. When I first saw her, the features were greatly distorted, the mouth pushed to one side, and her speech rendered imperfect, from a tumour as large as a moderate-sized orange, situated in the upper jaw, and which appeared to asise from expansion of the walls of the antrum. There was a considerable offensive discharge of sanious matter, and the tumour was exquisitely sensitive. From the size and hardness of the tumour, I at first thought it was a case of pure exostosis, but the result of treatment ultimately proved it to be one of periostosis.

I first extracted the diseased fangs, and freely lanced the surface of the tumour. I continued to lance the gum over the tumour every third or fourth day, for some weeks, and the result was always a copious discharge of pus and blood. The size of the tumour gradually diminished under this treatment, the fea- 